\title{
DAMPING IMPROVEMENT THROUGH TUNING CONTROLLER LIMITS OF A SERIES FACTS DEVICE
}

\author{
Jung-Wook Park $^{*}$ and Ian A. Hiskens ${ }^{* *}$ \\ * Core Technology Group, LG Electronics Inc., Seoul, South Korea \\ ** Department of Electrical and Computer Engineering, University of Wisconsin-Madison, USA \\ (Emails:jungwookpark@ieee.org and hiskens@engr.wisc.edu)
}

\begin{abstract}
The external controller (EC) of a series flexible ac transmission system (FACTS) device, which is the series capacitive reactance compensator (SCRC), can provide effective damping of lowfrequency oscillations in a power system. The saturation limiter in the EC is used to enforce practical limits resulting from the system restriction such as equipment and component ratings. In this paper, the effect of saturation limits on the overall system dynamic behavior is analyzed. Also, a systematic nonlinear tuning method is proposed to determine the optimal values for saturation limits.
\end{abstract}

\section{INTRODUCTION}

Rapid progress in power electronics has opened up new opportunities to control power and/or voltages, and this can be used to increase the capacities of existing power lines. In the last decade, the series flexible ac transmission systems (FACTS) devices have been progressively developed to deal with the above control objectives [1].

Among many power-electronic-based controllers that influence power flow in power lines, the controllable series capacitive reactance compensator (SCRC) using a voltage-source pulsewidth-modulated (PWM) inverter has attracted attention for its rapid response control capability to not only control the steady state, but also provide damping during disturbances [2]-[3]. Damping is also improved by applying external controller (EC) to the SCRC with the aid of properly designed supplementary controls [4].

The control outputs from the EC with high gains can cause undesirable response in a physical system. To enforce practical limits resulting from the system restriction, the saturation limiter following the EC is used. The overall power system structure, which has the SCRC, EC, and saturation limiter, is shown in Fig. 1 .

The optimal maximum $\left(\mathrm{EC}_{\max }\right)$ /minimum $\left(\mathrm{EC}_{\min }\right)$ values of saturation limiter in Fig. 1 can maximize stability immediately following large disturbance. The resulting non-smooth behavior is difficult to analyze, as discussed in [5] in the context of power system stabilizers (PSSs). Therefore, this study gives the framework of qualitatively assessing the outcomes of the various cases with saturation limits in the many industrial applications.
In this paper, the new contribution is made by analyzing the effects of the saturation limiter (with non-smooth nonlinearities) on system dynamics of a power network equipped with the SCRC device. Also, the nonlinear optimization method is proposed to determine the optimal values for saturation limits.

This paper is organized as follows: Section II presents a summary of the SCRC and EC. The effect of the saturation limiter on the system dynamic behavior is illustrated in Section III. The optimal tuning method for the saturation limits is described in Section IV. Finally, the conclusions are given in Section V.

\section{SCRC - SERIES FACTS DEVICE}

\subsection{Internal control for the SCRC}

The internal control scheme for the SCRC to drive the voltagesource PWM inverter is shown in Fig 1. The main objectives of inner control for the SCRC are to ensure that the injected voltage at ac terminals of inverter is in quadrature with transmission line current and to maintain the constant voltage $V_{d c}$ in steady state such as other series FACTS devices.

For the operation of the SCRC, the transmission line currents $i_{s a}$, $i_{s b}$, and $i_{s c}$ are first transformed by Park's transformation [1] into $d$ and $q$ axes components $i_{d}$ and $i_{q}$ in a synchronously rotating reference frame. Then, the peak value of the current vector is calculated. The desired magnitude of the compensating voltage vector is now determined by multiplying the magnitude of the current vector by the factor $2 \sqrt{2} / V_{d c}$ and the value of capacitive reactance $X_{C}$, and the result is the modulation index $m_{i}$ for the inverter.

Also, the structure of the SCRC in Fig. 1 has two feedback loops, which are an inner power control loop and an outer voltage control loop. The outer control loop ensures that no real power is exchanged between the inverter and the transmission line, thereby ensuring that the line current leads the injected voltage by $90^{\circ}$ as well as that the dc capacitor voltage $V_{d c}$ is kept constant. The inner feedback loop effectively reduces the gain required in the forward paths of internal control to achieve the overall closed loop bandwidth desired in the SCRC. 


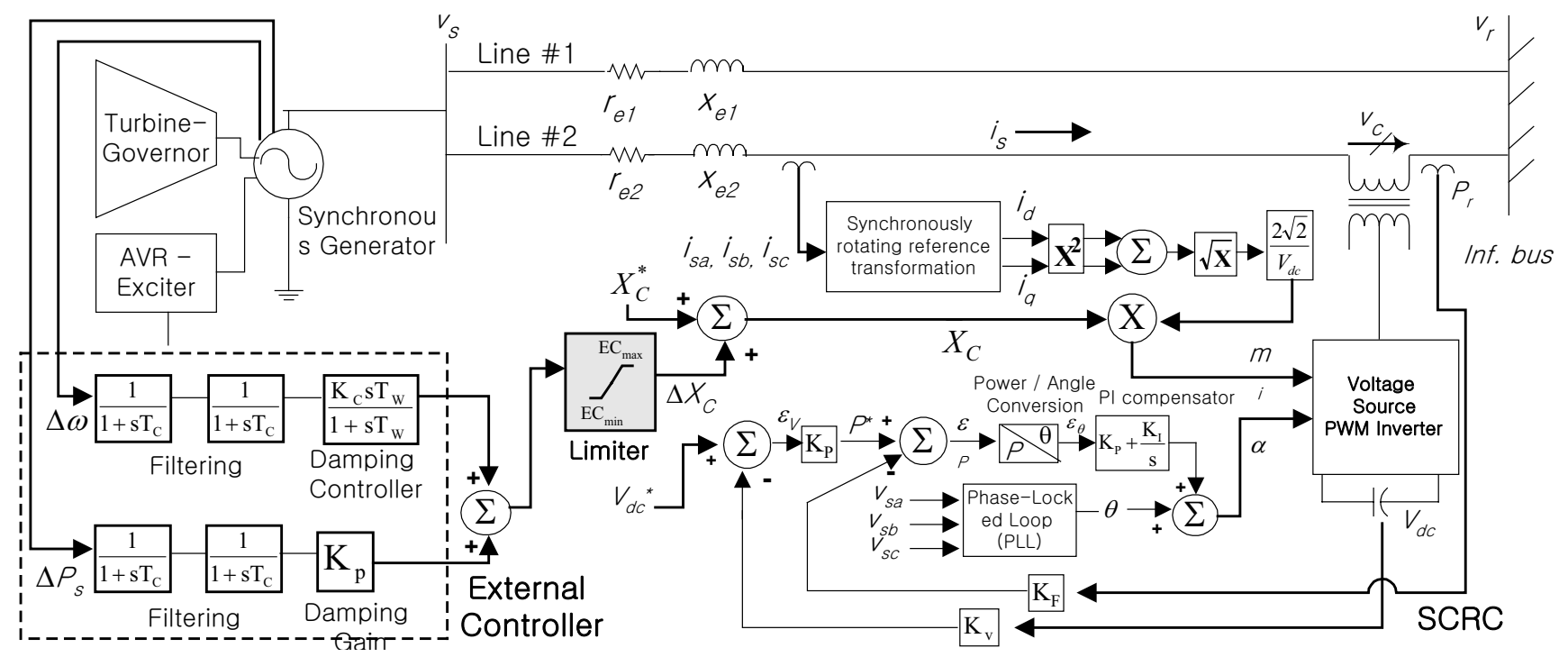

Figure 1. External controller (EC) and saturation limiter for the SCRC in a power system.

The advantage gained by the inclusion of the inner feedback loop is, therefore, that the necessary bandwidth of the phase-locked loop can be achieved with less compromise in the response of the system to a disturbance on the dc side of the inverter.

More details for the internal control of the SCRC are given in [3] and [4].

\subsection{External control for the SCRC}

The objective of the external controller (EC) for the SCRC (dashed block in Fig. 1) is to improve the system damping performance with the properly chosen external variables. It is suggested in [4] that the speed deviation signal $\Delta \omega$ and active power deviation signal $\Delta P_{S}$ (which exhibits the derivative behavior of the $\Delta \omega$ ) from a generator could be used to generate the supplementary control signal $\Delta X_{C}$ for the EC located close to that generator. Further explanation for the EC design is given in [4].

To design controller that satisfies the constraints of physical systems, the saturation limiter following the EC is now considered with non-smooth nonlinear dynamic behavior being the focus in this paper.

\subsection{Example system description}

The power system in Fig. 1 consists of the single generator (160 MVA, $15 \mathrm{kV}$ (L-L)) connected to an infinite bus through two transmission lines, labeled Line \#1 and Line \#2. The parameters of the synchronous generator are given in [6]. The EXAC1A (IEEE type alternator supplied rectifier excitation systems) and H_TUR1/GOV1 (IEEE type hydro turbine-governor) models in PSCAD/EMTDC ${ }^{\circledR}$ library [7] are used as the AVR/exciter and turbine/governor systems, respectively.

\section{EFFECT OF SATURATION LIMITS ON SYSTEM DYNAMICS}

The effect on the damping performance of the saturation limits is evaluated by a $100 \mathrm{~ms}$ three-phase short circuit applied at the infinite bus at $1 \mathrm{sec}$. The generator operates with a rotor angle of $16.38^{\circ}\left(P_{t}=0.25 \mathrm{pu}, Q_{t}=0.16 \mathrm{pu}\right)$ at a pre-fault steady state operating point, where the same amount of power is flowing in Line \#1 and Line \#2 (with the same line impedances).

The simulation results are given in Figs. 2 to 4 . It is clearly shown from Figs. 2 and 3 that the EC improves damping performance compared to the system without the EC. The corresponding EC output response $\left(\Delta X_{C}\right.$ [pu]) with non-smooth nonlinear dynamics due to saturation limits is shown in Fig. 4.

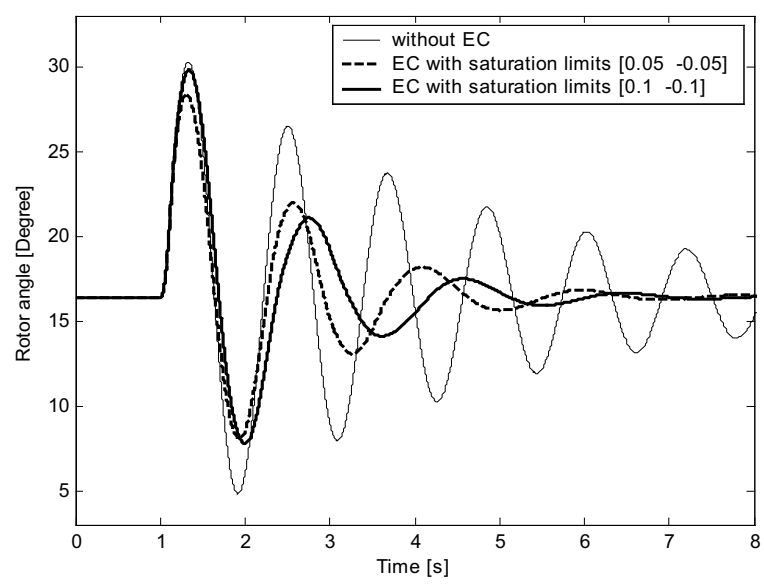

Figure 2. Rotor angle response $\left(\delta\left[^{\circ}\right]\right)$. 


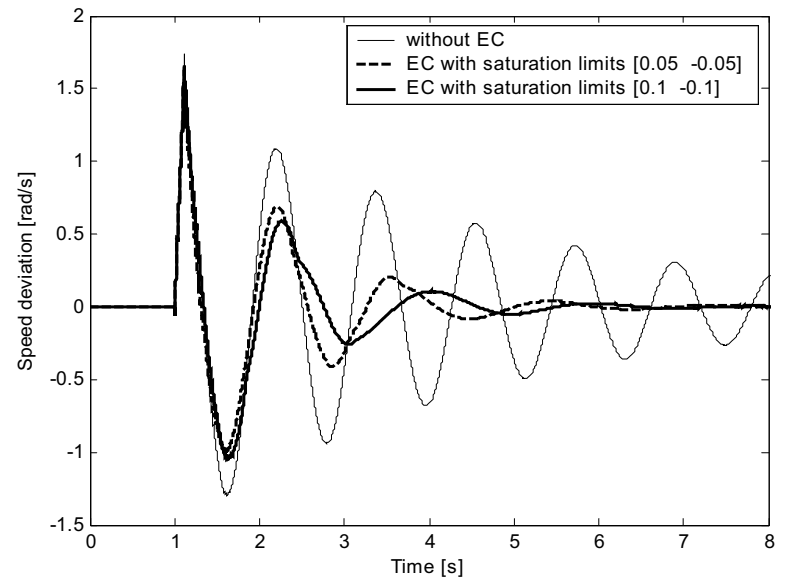

Figure 3. Speed deviation response $(\Delta \omega[\mathrm{rad} / \mathrm{s}])$.

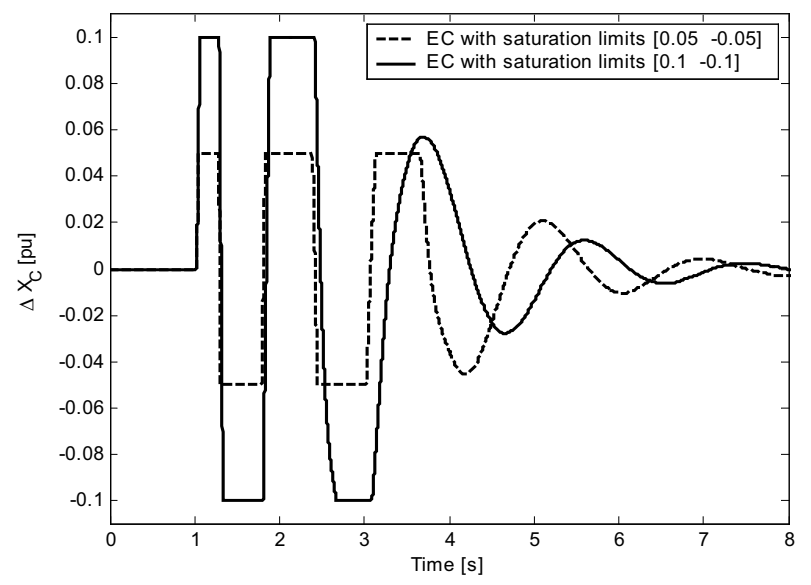

Figure 4. EC output response $\left(\Delta X_{C}[\mathrm{pu}]\right)$ : supplementary controls for the SCRC.

\section{OPTIMAL TUNING OF LIMTER}

\subsection{Optimization for non-smooth controller}

In multivariable nonlinear problems, numerical optimization methods play significant role in finding optimal solutions and selecting parameters by which the objective function $\mathbf{J}$ can be minimized/maximized. The optimal tuning problem for the limiter described in this paper is of this form.

The speed deviation $(\Delta \delta)$ and active power deviation $\left(\Delta P_{s}\right)$ from the generator, which are inputs of the EC, are considered good indicators of the damping. Therefore, damping improvement is equivalent to minimizing the objective function

$\mathbf{J}(\lambda)=\int_{t_{0}}^{t_{f}}\left(\left[\begin{array}{c}\omega(\lambda, t)-\omega^{*} \\ P_{s}(\lambda, t)-P_{s}^{*}\end{array}\right]^{T} \mathbf{W}\left[\begin{array}{c}\omega(\lambda, t)-\omega^{*} \\ P_{s}(\lambda, t)-P_{s}^{*}\end{array}\right]\right) d t$,

where $\mathbf{W}$ is the diagonal matrix of weighting factors and $t_{f}$ is an appropriate final time. The $\omega^{*}$ and $P_{S}{ }^{*}$ are the post-fault steady state values of $\omega$ and $P_{S}$, respectively. Minimization is achieved through variation of the saturation limits $\lambda=\left[\begin{array}{lll}\mathrm{EC}_{\max } & \mathrm{EC}_{\min }\end{array}\right]$.

\subsection{Computation of gradients}

Minimization of the nonlinear $\mathbf{J}$ in (1) requires knowledge of derivatives. Parameters $\lambda$ explicitly describe a non-smooth influence on behavior in a system that already involves switching devices of the voltage-source PWM inverter. Therefore, it is impossible to analytically determine the derivative of $\mathbf{J}$ with respect to parameters $\lambda$. However it has been shown in [8]-[9] that the required gradients, also known as trajectory sensitivities, are well defined for non-smooth, nonlinear systems. Furthermore, they can be efficiently computed as a by-product of numerical integration of the nominal trajectory.

Given the availability of gradient information, many numerical optimization methods based on the first-order gradients can be applied to minimize the function $\mathbf{J}$ in (1). The steepest descent method, which is the simplest first-order numerical optimization algorithm, is used to implement in this paper. The cost function in (1) has the form of a continuous-time nonlinear least-squares problem. A corresponding continuous-time adaptation of the Gauss-Newton or Broyden-Fletcher-Goldfarb-Shanno (BFGS) algorithms [10] is also appropriate, and can be efficiently applied.

\subsection{Results}

The damping performance achieved by the optimal saturation limits (which are $\left[\begin{array}{ll}0.0559 & -0.0971\end{array}\right]$ for $\left[\mathrm{EC}_{\max } \mathrm{EC}_{\min }\right]$ ) is compared with that of the initial output limits $\left(\left[\begin{array}{ll}0.05 & -0.05\end{array}\right]\right.$ and $\left.\left[\begin{array}{ll}0.1 & -0.1\end{array}\right]\right)$ by a $100 \mathrm{~ms}$ three-phase short circuit applied at the infinite bus in Fig. 1 at $1 \mathrm{sec}$.

The $\mathrm{EC}_{\max }$ value of 0.0559 has changed little from the initial positive limit value 0.05 , but $\mathrm{EC}_{\min }$ has moved from -0.05 to 0.0971 , which is close to -0.1 . The corresponding result is shown in Fig. 5. Note that the negative limit $\mathrm{EC}_{\min }$ is effective to damp the first swing, and the positive limit $\mathrm{EC}_{\max }$ improves damping performance after first swing (see the second swing).

Also, the values of the cost function $\mathbf{J}$ are compared in Table 1. The variations of the cost function $\mathbf{J}$ optimized during the 5 iterations are shown in Fig. 6.

\section{CONCLUSION}

This paper made the new contribution by applying the nonlinear optimization algorithm to tune the saturation limiter used in the external controller (EC) for the series capacitive reactance compensator (SCRC), which is a series flexible ac transmission system (FACTS) device. Trajectory sensitivities provided the derivatives of an objective function with respect to parameters that related directly to the non-smoothness of the limiter. The optimal limit values determined by the proposed method improved the overall system damping performance.

The systematic approach proposed in this paper to optimally tune the limiter can give a framework for industrial applications with saturation limits that affect the overall system dynamics. 


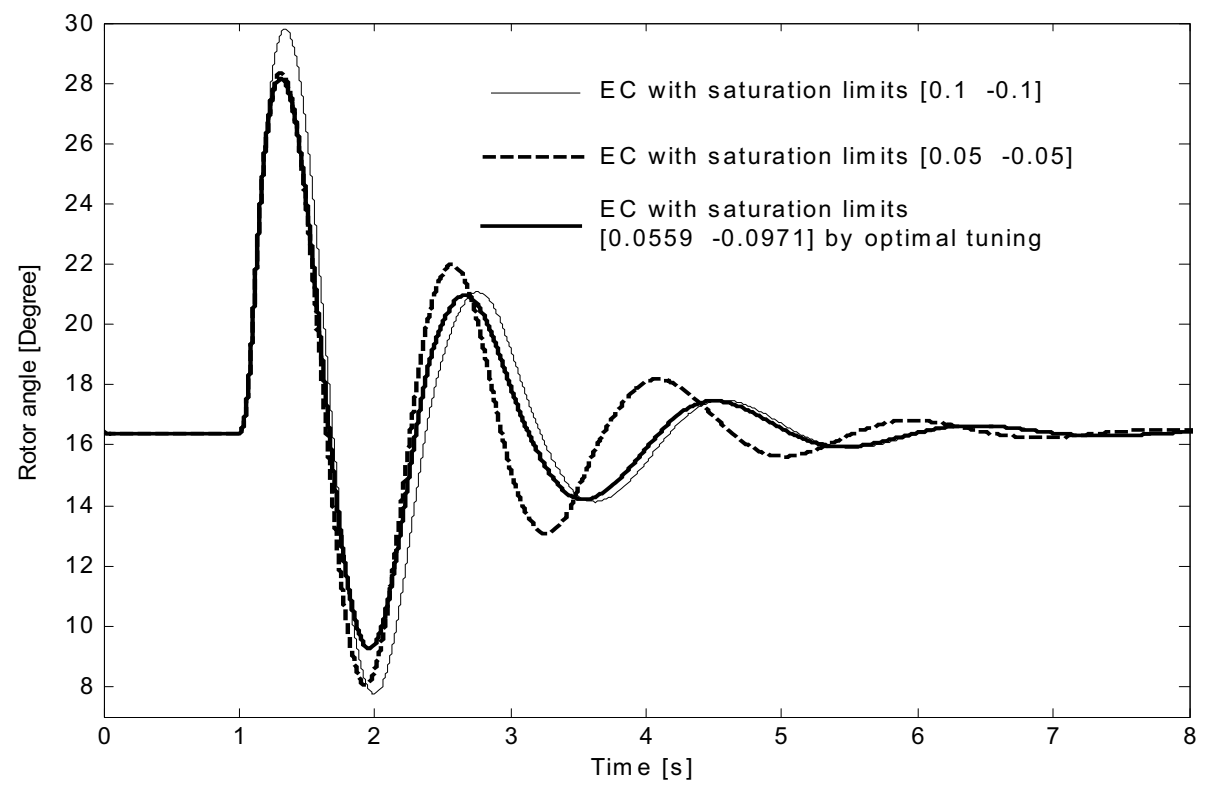

Figure 5. Rotor angle response $\left(\delta\left[^{\circ}\right]\right)$.

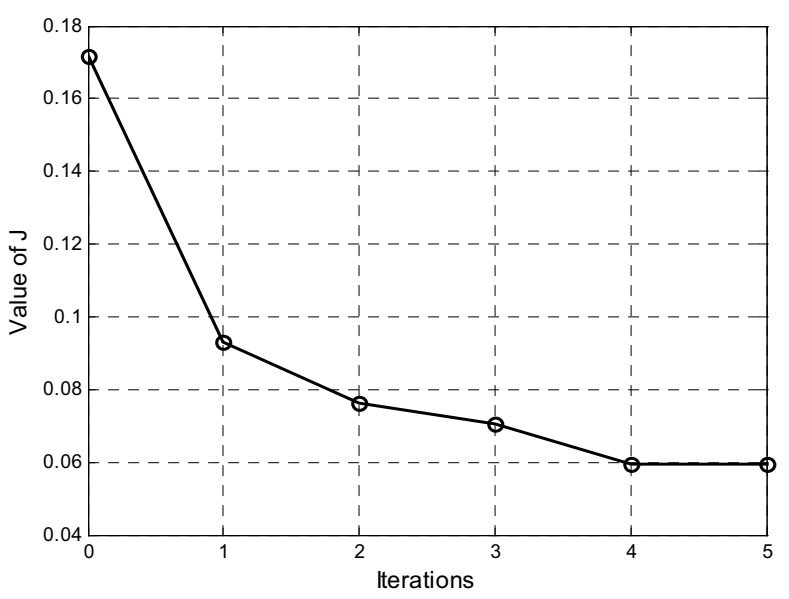

Figure 6. Values of the cost function $\mathbf{J}$ variations.

Table 1: Comparisons of the cost function $\mathbf{J}$

\begin{tabular}{|c|c|c|}
\hline$\left[\mathrm{EC}_{\max }\right.$ & $\left.\mathrm{EC}_{\text {min }}\right]$ & Cost, J \\
\hline$[0.05$ & $-0.05]$ & 0.1716 \\
\hline$[0.1$ & $-0.1]$ & 0.0847 \\
\hline$[0.0559$ & $-0.0971]$ & 0.0594 \\
\hline
\end{tabular}

\section{REFERENCES}

[1] N.G. Hingorani and L. Gyugyi, "Understanding FACTSConcepts and Technology of Flexible AC Transmission
Systems," IEEE Press, New York, 2000, ISBN 0-78033455-8.

[2] X. Wang, S.-Z. Dai, and B. T. Ooi, "A series capacitive reactance compensator based on voltage source PWM converter," in Conf. Rec. IEEE-IAS Annual Meeting, pp.918-924, 1991.

[3] Bruce S. Rigby and R.G. Harley, "An Improved control Scheme for a Series-Capacitive Reactance Compensator Based on a Voltage-Source Inverter," IEEE Trans. on Industry Applications, Vol.34, No.2, pp. 355-363, March/April 1998.

[4] Jung-Wook Park, R.G. Harley, and G.K. Venayagamoorthy, "New External Neuro-Controller for Series Capacitive Reactance Compensator in a Power Network," IEEE Trans. on Power Systems, Vol.19, No.3, pp. 1462-1472, August 2004.

[5] Ian A. Hiskens, "Systematic Tuning of Nonlinear Power System Controllers", in Proc. of IEEE International Conference on Control Applications, pp.19-24, September 2002.

[6] P.M. Anderson and A.A. Fouad, "Power system control and stability," IEEE Press, New York, 1994.

[7] Manitoba HVDC Research Centre Inc: "Introduction to PSCAD/EMTDC Version 4.2".

[8] Ian A. Hiskens and M.A. Pai, "Trajectory sensitivity analysis of hybrid systems," IEEE Trans. on Circuits and Systems I, Vol.47, No.2, pp. 204-220, February 2000.

[9] Ian A. Hiskens, "Power system modeling for inverse problems," IEEE Trans. on Circuits and Systems I: Regular Papers, Vol.51, No.3, pp. 539-551, March 2004.

[10] J. Nocedal and S. J. Wright, Numerical Optimization, Springer-Verlag, New York, 1999. 